\title{
A Fault Tolerant Control for Sensor and Actuator Failures of a Non Linear Hybrid System
}

\author{
Chalandi Hanene, Ben Slimane Jihane, Ksouri Moufida \\ University of Tunis El Manar, National School of Engineering of Tunis, Tunisia
}

\begin{tabular}{l} 
Article Info \\
\hline Article history: \\
Received Oct 15, 2017 \\
Revised Jan 31, 2018 \\
Accepted Feb 20, 2018 \\
\hline Keyword: \\
Active Fault Tolerant Control \\
Actuator \\
Hammerstein method. \\
Hybrid Systems \\
Reconfiguration \\
Sensor
\end{tabular}

Corresponding Author:

Chalandi Hanene,

\begin{abstract}
We focused in this work on a fault tolerant control of a non linear hybrid system class based on diagnosis method (determine and locate the defects and their types) and on the faults reconfiguration method. In literature we can found many important research activities over the fault-tolerant control of non linear systems and linear Hybrid systems. But it dosen't exist too many for the non linear hybrid system. The main idea in this paper is to consider a new approach to improve the reconfiguration performance of the non linear hybrid system by using hammerstein method which is designed to works only for linear systems. This method compensated the effect of the faults and guarantees the closed-loop system stable. The proposed method is simulated with a hydraulic system of two tanks with 4 modes
\end{abstract}

Copyright (C) 2018 Institute of Advanced Engineering and Science. All rights reserved.

University of Tunis El Manar, National School of Engineering of Tunis,

Laboratory of Research Analysis and Control of Systems (ACS) LR-11-ES20, Tunisia.

Email: h_chalandi@hotmail.fr

\section{INTRODUCTION}

In recent years, there has been important research activity in the fault-tolerant control for Hybrid systems. These systems contain continuous and discrete systems described by differential equations, automata and sequential logics. To control these systems even in case of failure, we adopted the fault tolerance control (FTC). The FTC is developed in two approaches: Passive approaches based on a robust controller that ensures the imperceptibility to the systems, and active approaches that change control operations for each detected fault [1], [2], [3].

In this paper, we are interested in a new control strategy for piecewise affine systems (PWA) that is used for system's reconfiguration. The contribution in this paper aims essentially to reconfigure the non linear hybrid system using the Hammerstein method that works only for linear systems. The linearization is obtained by using Taylor method.

The next sections in this paper are organized as follows: Section 2 presents the stability concepts, Section 3 describes the linearization, Section 4 discusses the reconfiguration with the Hammerstein method and Section 5 presents the simulation example.

\section{STABILITY NOTIONS}

In this part, we will present the stability technique with lyapunov method. Lyapunov method

To verify the stability of an hybrid system using the lyapunov method or more generally the LMI method. [3][4]. There exists a matrix

$$
P=P^{T}>0
$$


We say that the system is quadratically incrementally stable if it verifies the following LMI equation. [5] [6].

$$
P A_{i}+A_{i}^{T} P<0, i=1, \ldots, p
$$

\section{LINEARIZATION ( TAYLOR METHOD)}

If a Non Linear system has multiple dynamic variables on discrete time, it will be written as the following: [7], [8], [9], [10], [11], [12].

$$
\left(\begin{array}{l}
\dot{x}_{1} \\
\vdots \\
\dot{x}_{n}
\end{array}\right)=f(x, u)=\left(\begin{array}{l}
f_{1}\left(x_{1} \ldots x_{n}, u_{1} \ldots u_{m}\right) \\
\vdots \\
f_{n}\left(x_{1} \ldots x_{n}, u_{1} \ldots u_{m}\right)
\end{array}\right)
$$

where $\left(x_{1} \ldots x_{\mathrm{n}}\right)$ is the state vector and $\left(u_{1} \ldots u_{m}\right)$ is the control vector; $\mathrm{n}$ and $\mathrm{m}$ are positive integers.

When the system operates around an equilibrium point and the signals involved are small, then it is possible to approximate the Non Linear system with Taylor series by computing the Jacobian matrices in order to obtain a linear system with the following state space model:

$$
\left\{\begin{array}{l}
\dot{x}=A x+B u \\
y=C x+D u
\end{array}\right.
$$

Where $A$ is the state matrix, $B$ is the control input matrix, $C$ is the output matrix, $D$ is the feedthrough matrix and $y$ is the output vector. Now, we will approximate $f(x, u)$ with Taylor series:

$$
f(x, u)=f\left(x_{e}, u_{e}\right)+\left.\frac{\partial f(x, u)}{\partial x}\right|_{\left(x_{e}, u_{e}\right)}\left(x-x_{e}\right)+\left.\frac{\partial f(x, u)}{\partial u}\right|_{\left(x_{e}, u_{e}\right)}\left(u-u_{e}\right)
$$

Where $x_{e}$ and $u_{e}$ are the steady state operating points

$$
\begin{aligned}
& x_{e}=\left(\begin{array}{l}
x_{1 e} \\
\vdots \\
x_{n e}
\end{array}\right) \text { is a vector determined by solving: } \\
& \left\{\begin{array}{l}
\left.f_{1}\left(x_{1} \ldots x_{\mathrm{n}}, u_{1} \ldots u_{m}\right)\right|_{\left(x_{e}, u_{e}\right)}=x_{1 e} \\
\vdots \\
\left.f_{n}\left(x_{1} \ldots x_{\mathrm{n}}, u_{1} \ldots u_{m}\right)\right|_{\left(x_{e}, u_{e}\right)}=x_{n e}
\end{array}\right.
\end{aligned}
$$

To check the stability, it is necessary to find the Jacobian matrix:

$$
J=\frac{\partial f(x, u)}{\partial x}=\left[\begin{array}{ccc}
\frac{\partial f_{1}(x, u)}{\partial x_{1}} & \cdots & \frac{\partial f_{1}(x, u)}{\partial x_{n}} \\
\vdots & \ddots & \vdots \\
\frac{\partial f_{n}(x, u)}{\partial x_{1}} & \cdots & \frac{\partial f_{n}(x, u)}{\partial x_{n}}
\end{array}\right]
$$


that verifies:

$$
\operatorname{det}\left(J_{e}-\lambda I\right)=0
$$

Where: $J_{e}=\left.\frac{\partial f(x, u)}{\partial x}\right|_{\left(x_{e}, u_{e}\right)}$,

$\lambda=\left(\begin{array}{l}\lambda_{1} \\ \vdots \\ \lambda_{n}\end{array}\right)$ is the eigenvector, and $I$ is the identity matrix.

The equilibrium is locally stable if $(-1<\lambda<1)$.

Finally, we determine $y=g(x, u)$

$$
\begin{aligned}
& y=g\left(x_{e}, u_{e}\right)+\left.\frac{\partial g(x, u)}{\partial x}\right|_{\left(x_{e}, u_{e}\right)}\left(x-x_{e}\right)+\left.\frac{\partial g(x, u)}{\partial u}\right|_{\left(x_{e}, u_{e}\right)}\left(u-u_{e}\right) \\
& A=\left.\frac{\partial f(x, u)}{\partial x}\right|_{\left(x_{e}, u_{e}\right)}, B=\left.\frac{\partial f(x, u)}{\partial u}\right|_{\left(x_{e}, u_{e}\right)} \\
& C=\left.\frac{\partial g(x, u)}{\partial x}\right|_{\left(x_{e}, u_{e}\right)}, D=\left.\frac{\partial g(x, u)}{\partial u}\right|_{\left(x_{e}, u_{e}\right)}
\end{aligned}
$$

\section{RECONFIGURATION METHOD}

In this part, the piecewise affine system which is a class of a hybrid system is defined. Moreover, the reconfiguration method applied to hybrid system is processed.

\subsection{Piecewise Affine System (PWA)}

The piecewise affine system is characterized by dividing the state-space in a finite number of regions and associating to each one an affine linear equation. [13]. The nominal system is modeled by the following PWA model:

$$
\left\{\begin{array}{l}
\dot{x}(t)=A_{i} x(t)+b_{i}+B u_{c}(t)+B_{d} d(t) \\
y(t)=C x(t)
\end{array}\right.
$$

Where: $d(t)$ : disturbance

$\mathrm{B}_{\mathrm{d}}$ : disturbance Input matrix

$b_{i}$ : The affine terms

$A_{i}$ : the real matrices of appropriate dimensions for all $i$.

In this section the reconfiguration method is processed.

\subsection{Fault Model}

The fault event suddenly changes the nominal PWA system to the faulty PWA system:

$$
\left\{\begin{array}{l}
\dot{x}_{f}(t)=A_{i} x_{f}(t)+B_{f} u_{f}(t)+B_{d} d(t)+b_{i, f} \\
y_{f}(t)=C_{f} x_{f}(t)
\end{array}\right.
$$


Where: $B_{f}=$ corresponds to faulty actuator

$C_{f}=$ corresponds to faulty sensor

$u_{f}=$ corresponds to faulty controller

In case of actuator faults, we change the measurements of the matrix $B_{f}$ as follows:

$$
B_{f}=(1-\text { Beta }) \times B
$$

In case of Sensor faults, we change the measurements of matrix $C_{f}$ as follows:

$$
C_{f}=(\text { alpha }) \times C
$$

Beta, alpha are random values that keep the system faulty in the same interval of operation as the nominal system.

\subsection{Reconfiguration with Hammerstein Method}

The Hammerstein method is modeled by new input and output matrices of a system having actuator faults or sensor faults [14]. The reconfiguration with Hammerstein method is defined by the following equation:

1. In case of actuator faults, we obtain the following virtual actuator PWA:

$$
\left\{\begin{array}{l}
\dot{\tilde{x}}(t)=A_{i} \tilde{x}(t)+b_{i}+B_{\Delta, i} u_{c}(t) \\
y_{c}(t)=C \tilde{x}(t) \\
u_{f}(t)=M x(t)+N x(t)+B_{f}^{+} b_{i}
\end{array}\right.
$$

With

$$
B_{\Delta, i}= \pm\left(B-B_{f} M\right)
$$

$\tilde{X}$ is found by the following equation:

$$
\tilde{x}\left(A_{i}-B_{f} M\right)+\left(A_{i}-B_{f} M\right)^{T} \tilde{x}<0
$$

2. in case of a sensor fault, we obtain the following virtual sensor PWA:

$$
\left\{\begin{array}{l}
\dot{\hat{x}}_{f}(t)=A_{\delta, i} \hat{x}_{f}(t)+b_{i}+B u_{c}(t)+L y_{f}(t) \\
A_{\delta, i}=\left(A_{i}-L C_{f}\right)
\end{array}\right.
$$

With $\hat{X}$ is found by the following equation:

$$
\hat{x}_{f}\left(A_{i}-C_{f} L\right)+\left(A_{i}-C_{f} L\right)^{T} \hat{x}_{f}<0
$$

We find $\mathrm{M}$ and $\mathrm{L}$ with the following steps.

There exist matrices $X_{s}, Y_{s}$ and $X_{a}, Y_{a}$ that gratify the LMIs: 


$$
\begin{aligned}
& X_{s}=X_{s}^{T} \geq 0 \\
& Y_{s}=Y_{s}^{T} \geq 0 \\
& \bar{X}_{s} \bar{A}_{i}+\bar{A}_{i}^{T} \bar{X}_{s}-\bar{Y}_{s} \bar{C}_{f}-\bar{C}_{f}^{T} \bar{Y}_{s}^{T} \leq 0 \\
& \quad(i=1, \ldots, p)
\end{aligned}
$$

and

$$
\begin{aligned}
& X_{a}=X_{a}^{T} \geq 0 \\
& Y_{a}=Y_{a}^{T} \geq 0 \\
& A_{i} X_{a}+X_{a} A_{i}^{T}-B_{f} Y_{a}-Y_{a}^{T} B_{f}^{T} \leq 0 \\
& \quad(i=1, \ldots, p)
\end{aligned}
$$

$\mathrm{M}$ is a perturbation observer for the defective system: (for actuator fault)

$$
L \square X_{s}^{-1} Y_{s}
$$

The Hammerstein method is summarized in the following algorithm.

Steps 1 to 3 describe the nominal system before failure, in step 4 the defects are detected, the gains is calculated in step 5, in step 6 to 8 the reconfigured closed-loop system is run.

\begin{tabular}{|l|}
\hline \multicolumn{1}{|c|}{ Hammerstein Method } \\
\hline 1) request : PWA model $A_{i}, b_{i}, B, C$ \\
\hline 2) begin the nominal closed-loop system \\
$C_{f}=C, B_{f}=B, b_{f, i}=b_{i}, L=0, M=0$ \\
$N i=I, x(0)=x_{0}$ \\
\hline $\begin{array}{l}\text { 3) execution the nominal closed-loop system up to actuator or } \\
\text { sensor fault f detected and isolated }\end{array}$ \\
\hline 4) make the fault model $b_{f}, B_{f}, C_{f}$ and update the PWA (12) \\
\hline 5) find LMI (20),(21) and calculate (22) $M \square Y_{a} X_{a}^{-1}$ for \\
actuator fault, (23) $L \square X_{s}^{-1} Y_{s}$ for sensor fault \\
\hline $\begin{array}{l}\text { 6) calculate } B_{\Delta, j} \text { (16), } \tilde{x} \text { (17) for actuator fault, } \hat{x} \text { (19) for } \\
\text { sensor fault }\end{array}$ \\
\hline 7) Update PWA actuator fault (15), PWA sensor fault (18) \\
\hline 8) execution reconfigured closed-loop system \\
\hline
\end{tabular}

\section{EXPLICATIVE EXAMPLE} this section.

An application of Algorithm Hammerstein to the model of a two tanks with 4 modes is presented in

\subsection{The Non Linear Hybrid System}

In this part, we will present a model of two tanks with 4 modes as shown in Figure 1. The two-tank system is a typical example of a non linear hybrid system. It has been considered as a reference problem for the diagnosis and detection of failures in [15]. Table 1 shows the parameters of system: 


\begin{tabular}{ll} 
& \multicolumn{1}{c}{ Table 1. List of Symbols } \\
\hline \multicolumn{1}{c}{ Symbol } & Declaration \\
\hline $\mathrm{T}_{1}$ & Tank 1. \\
$\mathrm{T}_{2}$ & Tank 2. \\
$\mathrm{h}_{1}$ & liquid level in tank 1, initially 0.5m \\
$\mathrm{h}_{2}$ & liquid level in tank 2, initially empty \\
$\mathrm{P}$ & Pump controlled in all or none. \\
$\mathrm{C}_{1}, \mathrm{C}_{2}, \mathrm{C}_{3}, \mathrm{C}_{4}$ & Conducts. \\
$\mathrm{V}_{1}$ & Valve kept open during the functioning system \\
$\mathrm{V}_{2}$ & Valve kept open during the functioning system. \\
$\mathrm{V}_{4}$ & Controlled Valve $\left(\mathrm{V}_{4}\right.$ is opened at $\mathrm{t}=240 \mathrm{~s}$ and closed at $\left.\mathrm{t}=380 \mathrm{~s}\right)$. \\
$\mathrm{q}_{\mathrm{p}}$ & Debit in the pump $\mathrm{P},\left(\mathrm{q}_{\mathrm{p}}=0\right.$ when $\mathrm{h}_{2 \max }=0.2 \mathrm{~m}$ and $\mathrm{q}_{\mathrm{p}}=0.001 \mathrm{~m}^{3} / \mathrm{s}$ when $\left.\mathrm{h}_{2 \min }=0.1 \mathrm{~m}\right)$. \\
$\mathrm{q}_{1}$ & Debit in valve $\mathrm{V}_{1}$. \\
$\mathrm{q}_{2}$ & Debit in valve $\mathrm{V}_{2}$. \\
$\mathrm{q}_{3}$ & Transfer debit of conduct C3 at $0.5 \mathrm{~m} \mathrm{height.}$ \\
$\mathrm{q}_{4}$ & Debit in valve $\mathrm{V}_{4}$. \\
$\mathrm{S}$ & Section area of $\mathrm{T}_{1}$ and $\mathrm{T}_{2} ; \mathrm{S}=0.0154 \mathrm{~m}^{2}$. \\
$\mathrm{A}$ & Conducts Section; $\mathrm{A}=0.000036 \mathrm{~m}^{2}$. \\
\hline
\end{tabular}

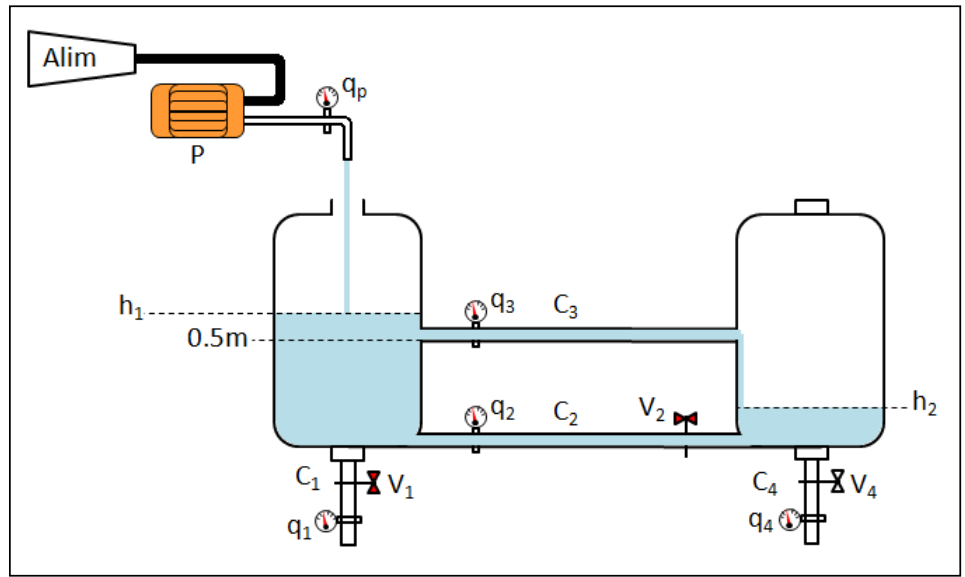

Figure 1. Two tanks system

The functioning of this system is described in Figure 2 by the automate hybrid.

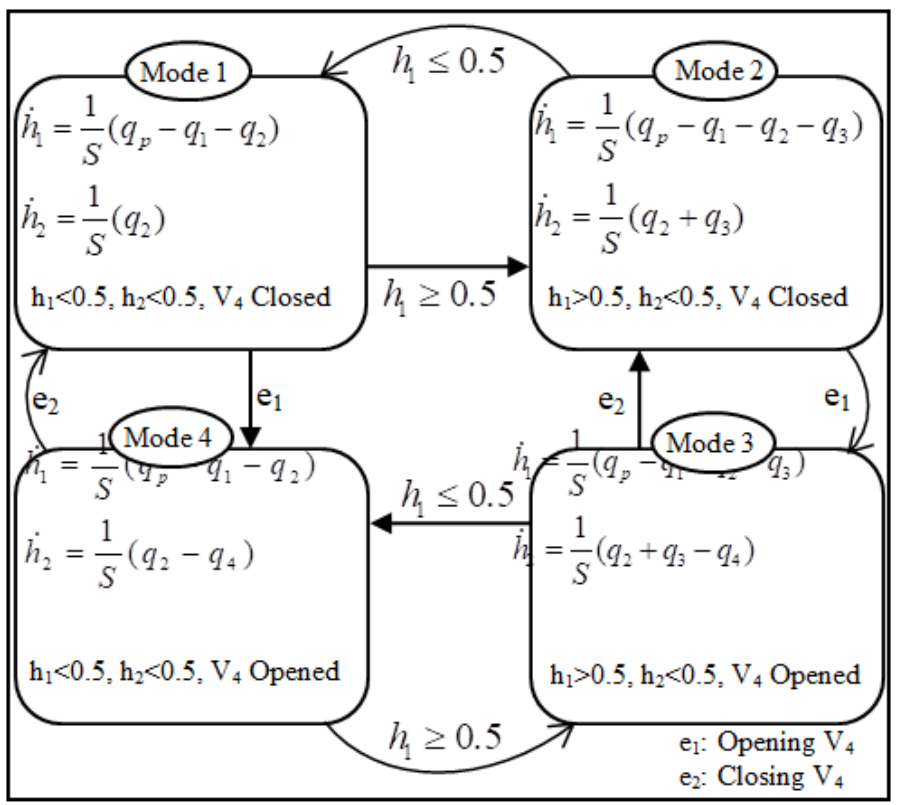

Figure 2. Automate hybrid 
The debits $q_{1}, q_{2}, q_{3}, q_{4}$ are given by the following equation:

$$
\left\{\begin{array}{l}
\mathrm{q}_{1}=A \sqrt{2 g h_{1}} \\
\mathrm{q}_{2}=\operatorname{sign}\left(\mathrm{h}_{1}-\mathrm{h}_{2}\right) \mathrm{A} \sqrt{2 \mathrm{~g}\left|\mathrm{~h}_{1}-\mathrm{h}_{2}\right|} \\
\mathrm{q}_{3}=\operatorname{sign}\left(\mathrm{h}_{1}-0.5\right) \mathrm{A} \sqrt{2 \mathrm{~g}\left|\mathrm{~h}_{1}-0.5\right|} \\
\mathrm{q}_{4}=\mathrm{A} \sqrt{2 \mathrm{gh}_{2}}
\end{array}\right.
$$

Note that the system is discretized with a sampling period $T_{e}=0.5 \mathrm{~s}$.

Figure 3 represents the liquid level variation of the non linear System.

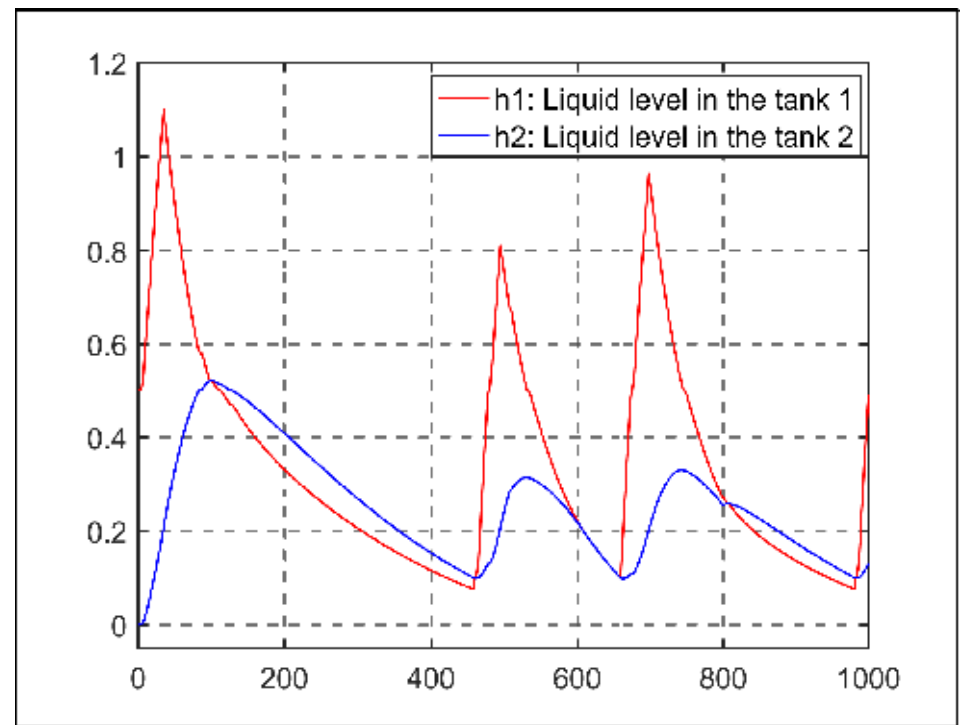

Figure 3. Liquid level variation of the non linear system

In this paper, we are interested in the reconfiguration of the non linear hybrid system. Whereas, the Hammerstein method is valid only for linear systems. We can summarized the work as: (1) providing an linearization method for a non linear Hybrid Systems ;(2) developing a Hammerstein algorithm which addresses both of the actuator faults and the sensor faults.

\subsection{Linearization of the non Linear Hybrid System}

This method of linearization is applied to our hybrid system:

$$
\begin{aligned}
& A=\left(\begin{array}{ll}
\frac{\partial \dot{h}_{1}}{\partial h_{1}} & \frac{\partial \dot{h}_{1}}{\partial h_{2}} \\
\frac{\partial \dot{h}_{2}}{\partial h_{1}} & \frac{\partial \dot{h}_{2}}{\partial h_{2}}
\end{array}\right), \quad B=\left(\begin{array}{ll}
\frac{\partial \dot{h}_{1}}{\partial q_{p}} & \frac{\partial \dot{h}_{1}}{\partial q_{4}} \\
\frac{\partial \dot{h}_{2}}{\partial q_{p}} & \frac{\partial \dot{h}_{2}}{\partial q_{4}}
\end{array}\right) \\
& C=\left(\begin{array}{ll}
1 & 0 \\
0 & 1
\end{array}\right), \quad D=\left(\begin{array}{ll}
0 & 0 \\
0 & 0
\end{array}\right), \quad x=\left(\begin{array}{l}
h_{1} \\
h_{2}
\end{array}\right), \quad u=\left(\begin{array}{l}
q_{p} \\
q_{4}
\end{array}\right)
\end{aligned}
$$

Matrices searching process for each mode:

1. Mode 1: $h_{1}<0.5, h_{2}<0.5, V_{4}$ Closed 
The system's equations will be written as follows:

$$
\begin{aligned}
& \left\{\begin{array}{l}
\dot{h}_{1}=\frac{1}{S}\left(q_{p}-q_{1}-q_{2}\right) \\
\dot{h}_{2}=\frac{1}{S}\left(q_{2}\right)
\end{array}\right. \\
& \left\{\begin{array}{l}
\dot{h}_{1}=\frac{1}{S}\left(q_{p}-A \sqrt{2 g h_{1}}-\operatorname{sgn}\left(h_{1}-h_{2}\right) A \sqrt{2 g\left|h_{1}-h_{2}\right|}\right) \\
\dot{h}_{2}=\frac{1}{S}\left(\operatorname{sgn}\left(h_{1}-h_{2}\right) A \sqrt{2 g\left|h_{1}-h_{2}\right|}\right)
\end{array}\right.
\end{aligned}
$$

The steady state operating points $h_{1 e}$ and $h_{2 e}$ are given by solving:

$$
\left\{\begin{array}{l}
\left.\dot{h}_{1}\right|_{\left(h_{1 e}, h_{2 e}\right)}=h_{1 e} \\
\left.\dot{h}_{2}\right|_{\left(h_{1 e}, h_{2 e}\right)}=h_{2 e}
\end{array}\right.
$$

The Jacobian matrix:

$$
J=\left(\begin{array}{ll}
\frac{\partial \dot{h}_{1}}{\partial h_{1}} & \frac{\partial \dot{h}_{1}}{\partial h_{2}} \\
\frac{\partial \dot{h}_{2}}{\partial h_{1}} & \frac{\partial \dot{h}_{2}}{\partial h_{2}}
\end{array}\right)=\frac{A \sqrt{2 g}}{2 s}\left(\begin{array}{cc}
-\frac{1}{\sqrt{h_{1}}}-\frac{1}{\sqrt{\left|h_{1}-h_{2}\right|}} & \frac{1}{\sqrt{\left|h_{1}-h_{2}\right|}} \\
\frac{1}{\sqrt{\left|h_{1}-h_{2}\right|}} & -\frac{1}{\sqrt{\left|h_{1}-h_{2}\right|}}
\end{array}\right)
$$

The Jacobian matrix at the operating points $h_{1 e}$ and $h_{2 e}$ :

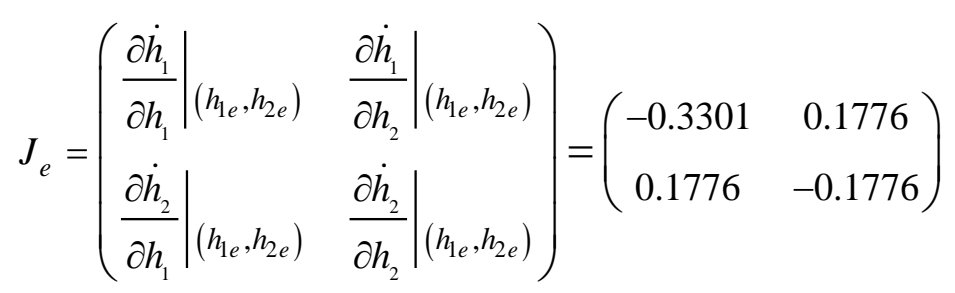

Calculating the eigenvector of $J_{e}$ :

$$
\lambda=\left(\begin{array}{l}
\lambda_{1} \\
\lambda_{2}
\end{array}\right)=\left(\begin{array}{l}
-0.0606 \\
-0.4471
\end{array}\right)
$$

$\left(-1<\lambda_{1}<1\right)$ and $\left(-1<\lambda_{2}<1\right)$ so the equilibrium is locally stable, then $A=J_{e}$. 
The control matrix $B$ is calculated as follows:

$$
B=\left(\begin{array}{ll}
\frac{\partial \dot{h}_{1}}{\partial q_{p}} & \frac{\partial \dot{h}_{1}}{\partial q_{4}} \\
\frac{\partial \dot{h}_{2}}{\partial q_{p}} & \frac{\partial \dot{h}_{2}}{\partial q_{p}}
\end{array}\right)=\left(\begin{array}{cc}
\frac{1}{S} & 0 \\
0 & 0
\end{array}\right)
$$

2. Mode 2: $h_{1}>0.5, h_{2}<0.5, V_{4}$ Closed

The system's equations will be written as follows:

$$
\begin{aligned}
& \left\{\begin{array}{l}
\dot{h}_{1}=\frac{1}{S}\left(q_{p}-q_{1}-q_{2}-q_{3}\right) \\
\dot{h}_{2}=\frac{1}{S}\left(q_{2}+q_{3}\right)
\end{array}\right. \\
& \left\{\begin{array}{l}
\dot{h}_{1}=\frac{1}{S}\left(q_{p}-A \sqrt{2 g h_{1}}-\operatorname{sgn}\left(h_{1}-h_{2}\right) A \sqrt{2 g\left|h_{1}-h_{2}\right|}-\operatorname{sgn}\left(h_{1}-0.5\right) A \sqrt{2 g\left|h_{1}-0.5\right|}\right) \\
\dot{h}_{2}=\frac{1}{S}\left(\operatorname{sgn}\left(h_{1}-h_{2}\right) A \sqrt{2 g\left|h_{1}-h_{2}\right|}+\operatorname{sgn}\left(h_{1}-0.5\right) A \sqrt{2 g\left|h_{1}-0.5\right|}\right)
\end{array}\right.
\end{aligned}
$$

The Jacobian matrix:

$$
J=\frac{A \sqrt{2 g}}{2 s}\left(\begin{array}{cc}
-\frac{1}{\sqrt{h_{1}}}-\frac{1}{\sqrt{\left|h_{1}-h_{2}\right|}}-\frac{1}{\sqrt{\left|h_{1}-0.5\right|}} & \frac{1}{\sqrt{\left|h_{1}-h_{2}\right|}} \\
\frac{1}{\sqrt{\left|h_{1}-h_{2}\right|}+\frac{1}{\sqrt{\left|h_{1}-0.5\right|}}} & -\frac{1}{\sqrt{\left|h_{1}-h_{2}\right|}}
\end{array}\right)
$$

The Jacobian matrix at the operating points:

$$
J_{e}=\left(\begin{array}{cc}
-0.1259 & 0.0481 \\
0.0555 & -0.0481
\end{array}\right)
$$

Calculating the eigenvector of $J_{e}$ :

$$
\lambda=\left(\begin{array}{l}
\lambda_{1} \\
\lambda_{2}
\end{array}\right)=\left(\begin{array}{l}
-0.0223 \\
-0.1517
\end{array}\right)
$$

$(-1<\lambda<1)$, so the equilibrium is locally stable, then $A=J_{e}$.

The control matrix $B$ is calculated as follows:

$$
B=\left(\begin{array}{cc}
\frac{1}{S} & 0 \\
0 & 0
\end{array}\right)
$$


3. Mode 3: $h_{1}>0.5, h_{2}<0.5, V_{4}$ Opened

The system's equations will be written as follows:

$$
\begin{aligned}
& \left\{\begin{array}{l}
\dot{h}_{1}=\frac{1}{S}\left(q_{p}-q_{1}-q_{2}-q_{3}\right) \\
\dot{h}_{2}=\frac{1}{S}\left(q_{2}+q_{3}-q_{4}\right)
\end{array}\right. \\
& \left\{\begin{array}{l}
\dot{h}_{1}=\frac{1}{S}\left(q_{p}-A \sqrt{2 g h_{1}}-\operatorname{sgn}\left(h_{1}-h_{2}\right) A \sqrt{2 g\left|h_{1}-h_{2}\right|}-\operatorname{sgn}\left(h_{1}-0.5\right) A \sqrt{2 g\left|h_{1}-0.5\right|}\right) \\
\dot{h}_{2}=\frac{1}{S}\left(\operatorname{sgn}\left(h_{1}-h_{2}\right) A \sqrt{2 g\left|h_{1}-h_{2}\right|}+\operatorname{sgn}\left(h_{1}-0.5\right) A \sqrt{2 g\left|h_{1}-0.5\right|}-q_{4}\right)
\end{array}\right.
\end{aligned}
$$

The Jacobian matrix:

$$
J=\frac{A \sqrt{2 g}}{2 s}\left(\begin{array}{cc}
-\frac{1}{\sqrt{h_{1}}}-\frac{1}{\sqrt{\left|h_{1}-h_{2}\right|}}-\frac{1}{\sqrt{\left|h_{1}-0.5\right|}} & \frac{1}{\sqrt{\left|h_{1}-h_{2}\right|}} \\
\frac{1}{\sqrt{\left|h_{1}-h_{2}\right|}+\frac{1}{\sqrt{\left|h_{1}-0.5\right|}}} & -\frac{1}{\sqrt{\left|h_{1}-h_{2}\right|}}
\end{array}\right)
$$

The Jacobian matrix at the operating points $h_{1 e}$ and $h_{2 e}$ :

$$
J_{e}=\left(\begin{array}{cc}
-0.1191 & 0.0399 \\
0.0473 & -0.0399
\end{array}\right)
$$

Calculating the eigenvector of $J_{e}$ :

$$
\lambda=\left(\begin{array}{l}
\lambda_{1} \\
\lambda_{2}
\end{array}\right)=\left(\begin{array}{l}
-0.0207 \\
-0.1383
\end{array}\right)
$$

$(-1<\lambda<1)$, so the equilibrium is locally stable, then $A=J_{e}$.

The control matrix $B$ is calculated as follows:

$$
B=\left(\begin{array}{cc}
\frac{1}{S} & 0 \\
0 & -\frac{1}{S}
\end{array}\right)
$$

4. Mode 4: $h_{1}<0.5, h_{2}<0.5, V_{4}$ Opened

The system's equations will be written as follows:

$$
\left\{\begin{array}{l}
\dot{h}_{1}=\frac{1}{S}\left(q_{p}-q_{1}-q_{2}\right) \\
\dot{h}_{2}=\frac{1}{S}\left(q_{2}-q_{4}\right)
\end{array}\right.
$$




$$
\left\{\begin{array}{l}
\dot{h}_{1}=\frac{1}{S}\left(q_{p}-A \sqrt{2 g h_{1}}-\operatorname{sgn}\left(h_{1}-h_{2}\right) A \sqrt{2 g\left|h_{1}-h_{2}\right|}\right) \\
\dot{h}_{2}=\frac{1}{S}\left(\operatorname{sgn}\left(h_{1}-h_{2}\right) A \sqrt{2 g\left|h_{1}-h_{2}\right|}-q_{4}\right)
\end{array}\right.
$$

The Jacobian matrix:

$$
J=\frac{A \sqrt{2 g}}{2 s}\left(\begin{array}{cc}
-\frac{1}{\sqrt{h_{1}}-\frac{1}{\sqrt{\left|h_{1}-h_{2}\right|}}} & \frac{1}{\sqrt{\left|h_{1}-h_{2}\right|}} \\
\frac{1}{\sqrt{\left|h_{1}-h_{2}\right|}} & -\frac{1}{\sqrt{\left|h_{1}-h_{2}\right|}}
\end{array}\right)
$$

The Jacobian matrix at the operating points:

$$
J_{e}=\left(\begin{array}{cc}
-0.2418 & 0.0681 \\
0.0681 & -0.0681
\end{array}\right)
$$

Calculating the eigenvector of $J_{e}$ :

$$
\lambda=\left(\begin{array}{l}
\lambda_{1} \\
\lambda_{2}
\end{array}\right)=\left(\begin{array}{l}
-0.0446 \\
-0.2654
\end{array}\right)
$$

$(-1<\lambda<1)$, so the equilibrium is locally stable, then $A=J_{e}$.

The control matrix $B$ is calculated as follows:

$$
B=\left(\begin{array}{cc}
\frac{1}{s} & 0 \\
0 & -\frac{1}{s}
\end{array}\right)
$$

\subsection{Reconfiguration method}

The adopted reconfiguration method applied to hybrid system is processed in this section. In the first part, an actuator fault is introduced to the system and the performance of the Hammerstein method by reconfiguration of the actuator fault is checked. In the second part, a sensor fault is inserted to the system and then a reconfiguration of a sensor fault is undertaken.

\subsubsection{Actuator Fault and Reconfiguration:}

a. Actuator Fault model:

The new matrix $B_{f}$ equals:

$$
B_{f}=(1-B e t a) \times B
$$


Figure 4 represents the linear system and the actuator fault system.

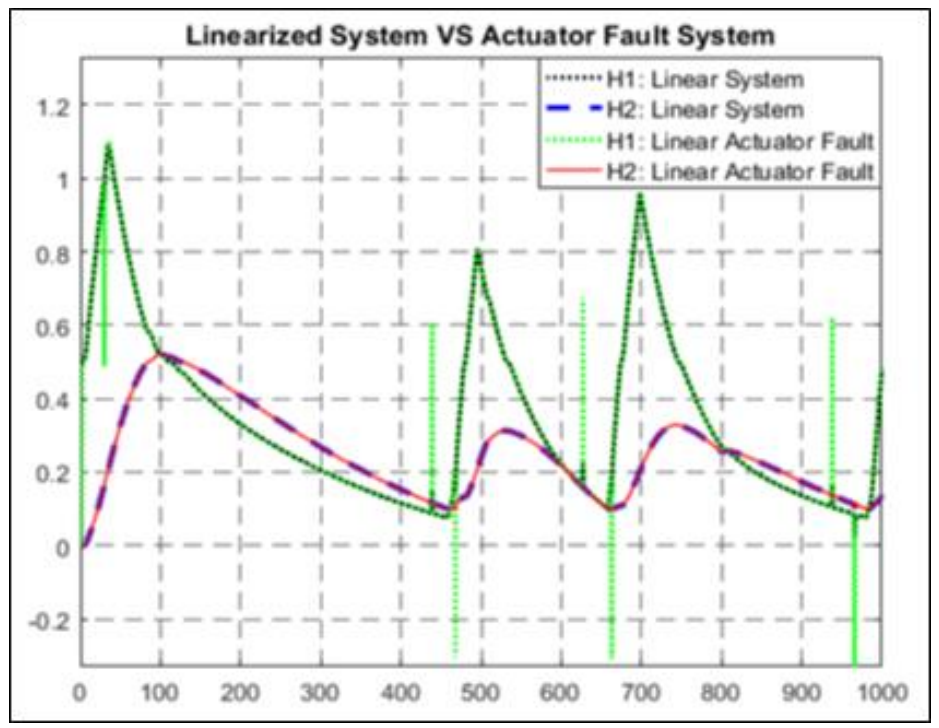

Figure 4. System with actuator fault

Figure 5 is Figure 4 focused on the fault part of the actuator fault system.

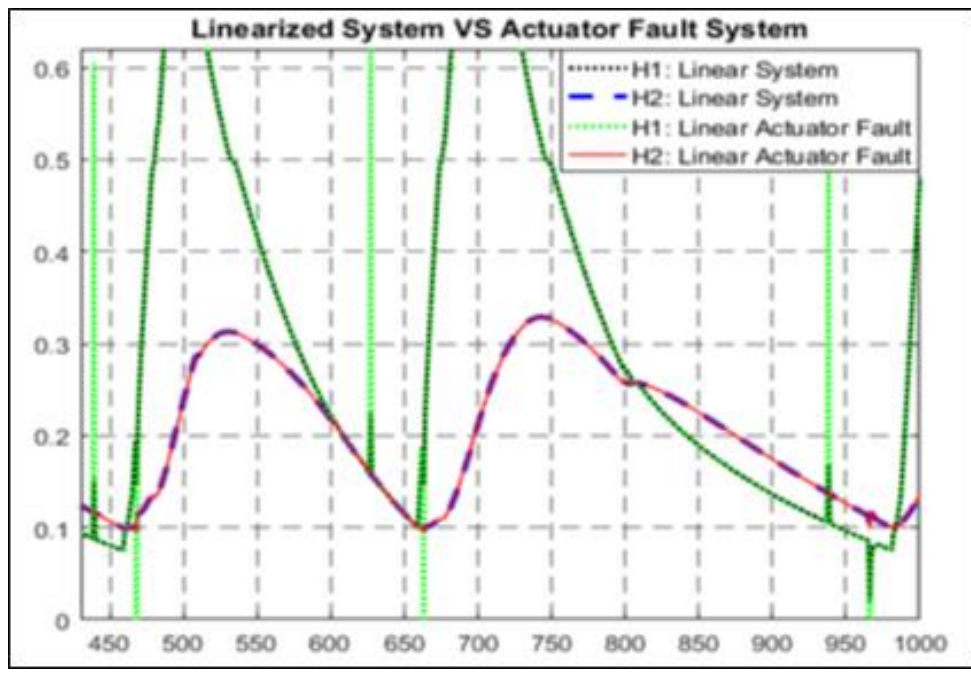

Figure 5. System with actuator fault (Zoom)

Failures detection is neatly visible on the curves of Figures 4 and 5, the fault is introduced during the time interval [400-1000s], the failure is detected at $t=460 \mathrm{~s}, \mathrm{t}=660 \mathrm{~s}$, and $\mathrm{t}=982 \mathrm{~s}$.

b. Reconfiguration of the Actuator Fault by Hammerstein method:

We find $M$ (perturbation observer):

$$
M \square Y_{a} X_{a}^{-1}=10^{-3}\left[\begin{array}{rr}
-0.4214 & -0.5342 \\
0.1500 & -0.5717
\end{array}\right]
$$

$\tilde{X}$ Is found by the following equation:

$$
\tilde{x}\left(A_{i}-B_{f} M\right)+\left(A_{i}-B_{f} M\right)^{T} \tilde{x}<0
$$




$$
\tilde{x}=\left[\begin{array}{ll}
0.0069 & 0.1053 \\
0.1053 & 0.1676
\end{array}\right]
$$

Figure 6 represents the reconfiguration for an actuator fault system.

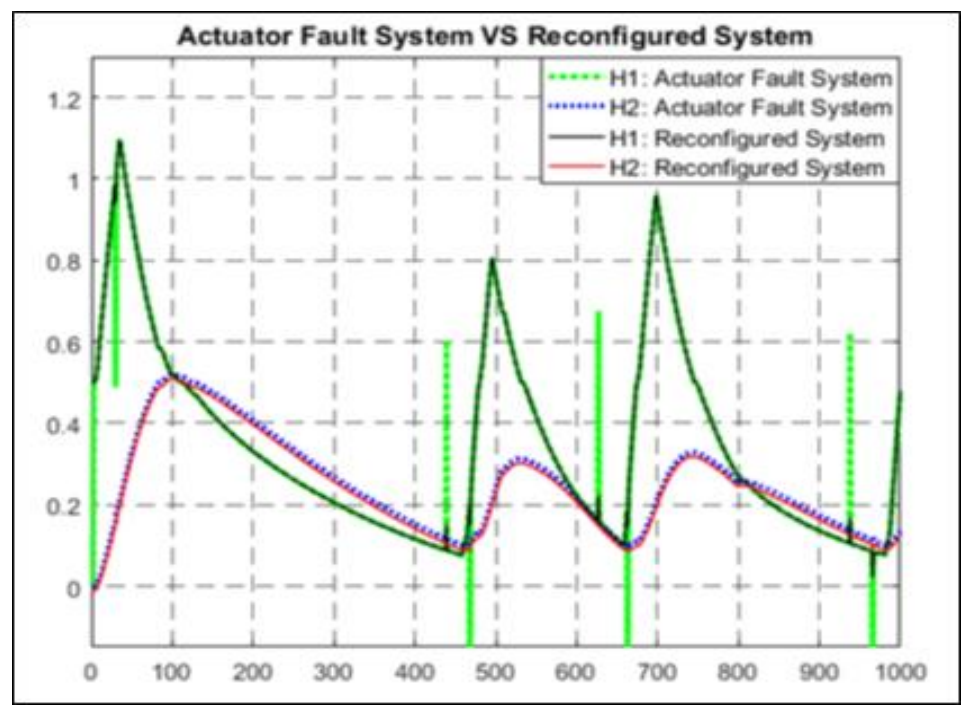

Figure 6. Reconfiguration for an actuator fault

Figure 7 shows the Comparison of the linear system with the reconfiguration system for an actuator fault.

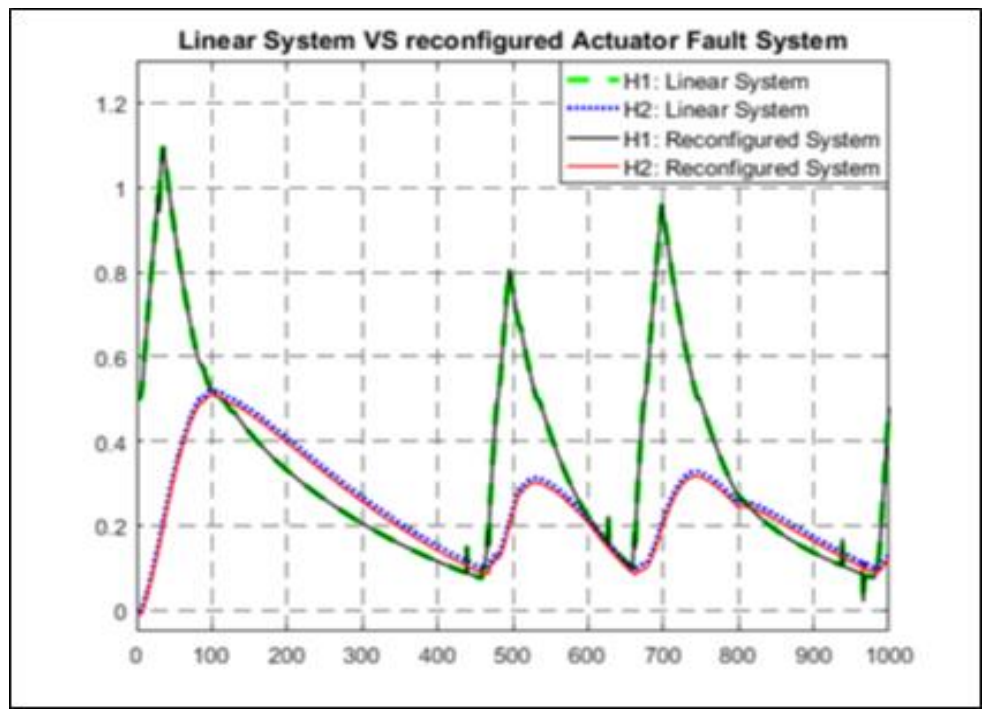

Figure 7. Comparison of the linear system with the reconfiguration system for an actuator fault

Figure 8 is a zoomed version of the Figure 7; it shows that the adopted method has successfully compensate the effect of the faults on the system. 


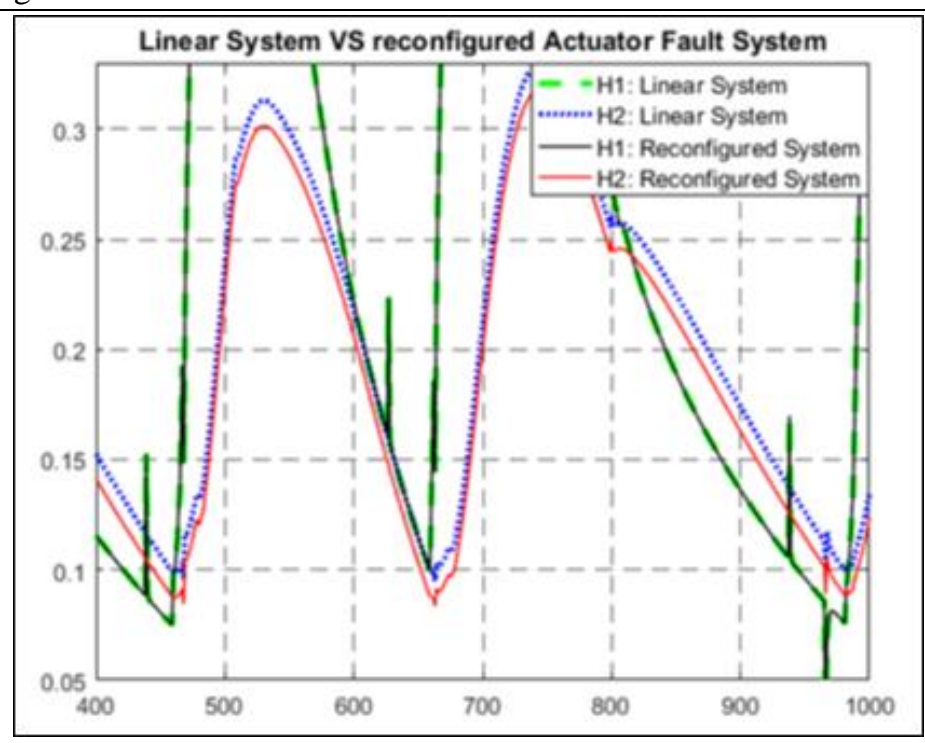

Figure 8. Comparison of the linear system with the reconfiguration system for an actuator fault (Zoom)

Figure 9 shows the efficiency of the reconfiguration with Hammerstein method for an actuator fault.

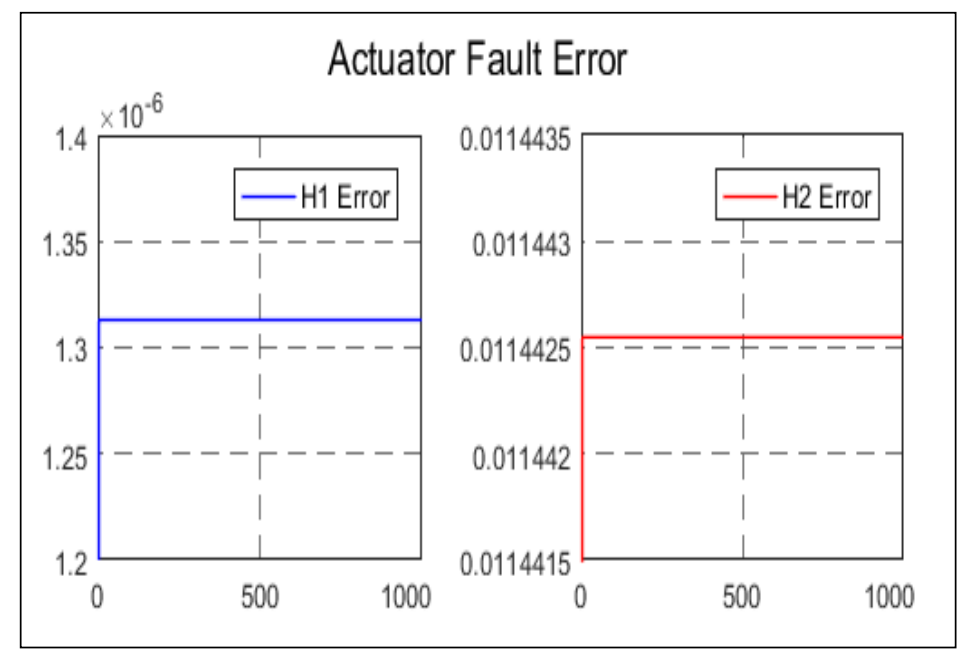

Figure 9. Error between the linear system and the reconfiguration system for an actuator fault

The computation of the error is realized for a time period between the linear system and the reconfiguration system for an actuator fault. It is interesting to note that the error's value is close to zero. From the previous results, the figures show that the Hammerstein method is very effective to reconfigured the actuator faults.

\subsubsection{Sensor Fault and Reconfiguration:}

a. Sensor Fault model:

The new matrix $\mathrm{C}_{\mathrm{f}}$ equals:

$$
C_{f}=(\text { alpha }) \times C=\text { alpha }\left[\begin{array}{ll}
1 & 0 \\
0 & 1
\end{array}\right]
$$


Figure 10 represents the linear system and the sensor fault system

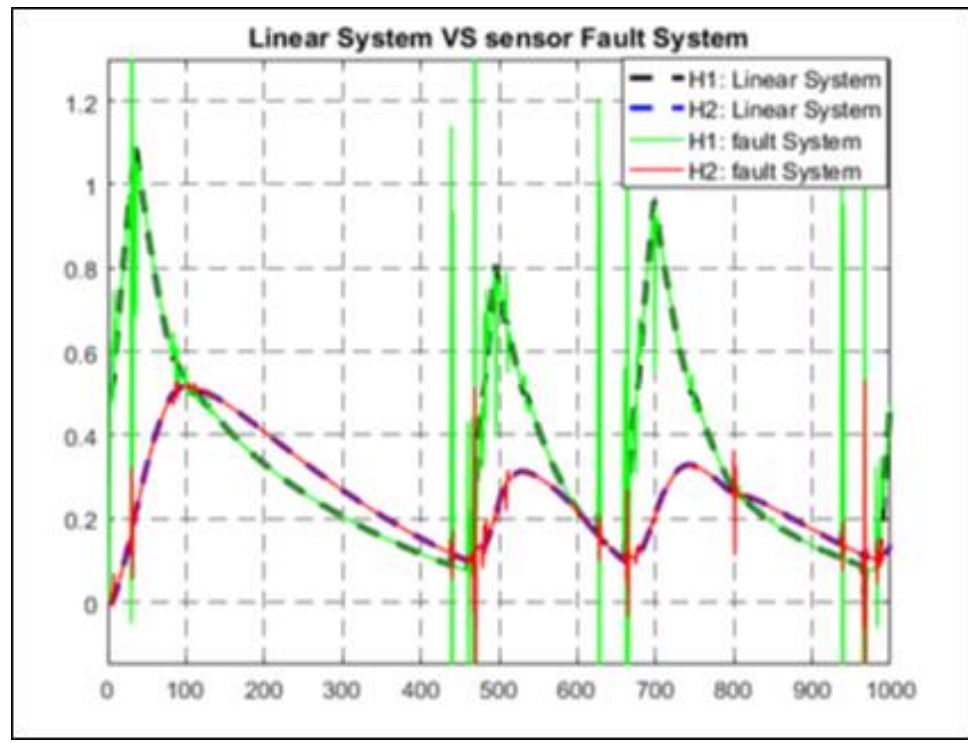

Figure 10. System with sensor fault

Figure 11 is a zoomed version of the Figure 10.

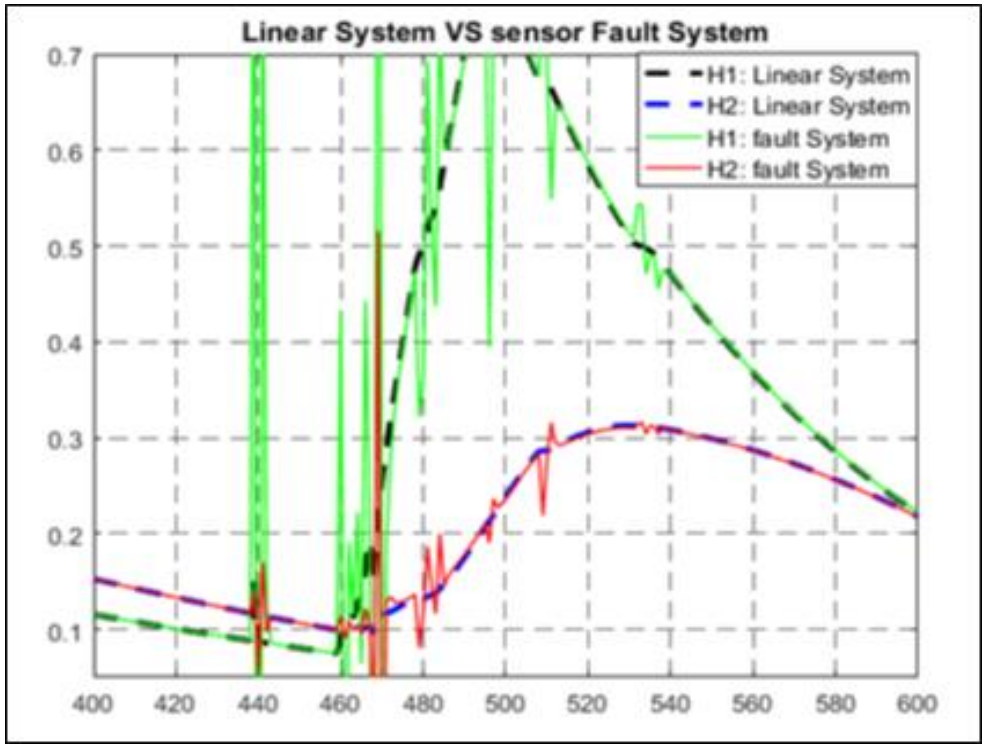

Figure 11. System with sensor fault (Zoom)

Failures detection is neatly visible on the figures 10 and 11, the fault is introduced during the time interval [400-1000s], the failure is detected at $\mathrm{t}=440 \mathrm{~s}, \mathrm{t}=460 \mathrm{~s}, \mathrm{t}=475 \mathrm{~s}, \mathrm{t}=490 \mathrm{~s}$, and $\mathrm{t}=533 \mathrm{~s}$.

b. Reconfiguration of the Sensor Fault by Hammerstein method:

We find L (state observer)

$$
L \square X_{s}^{-1} Y_{s}=-0.2274
$$


With $\hat{X}$ is found by the following equation:

$$
\begin{aligned}
& \hat{x}_{f}\left(A_{i}-C_{f} L\right)+\left(A_{i}-C_{f} L\right)^{T} \hat{x}_{f}<0 \\
& \tilde{x}=10^{-3}\left[\begin{array}{ll}
0.8099 & 0.0108 \\
0.0108 & 0.8126
\end{array}\right]
\end{aligned}
$$

Figure 12 represents the reconfiguration for a sensor fault system.

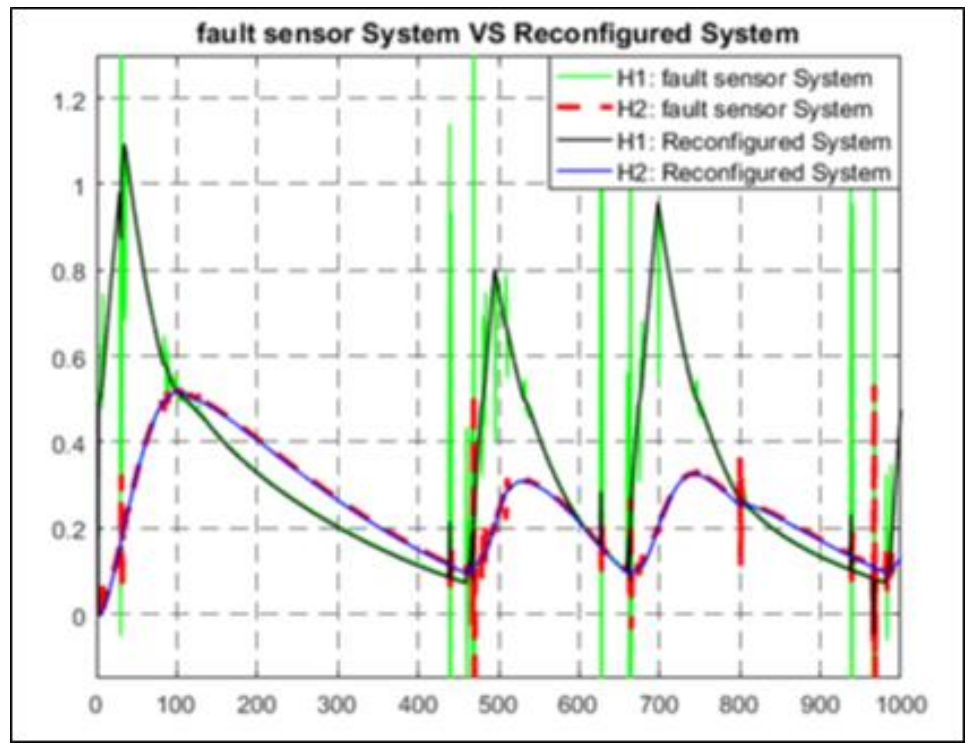

Figure 12. Reconfiguration for a sensor fault

Figure 13 represents the comparison between the linear system and the reconfiguration system for a sensor fault.

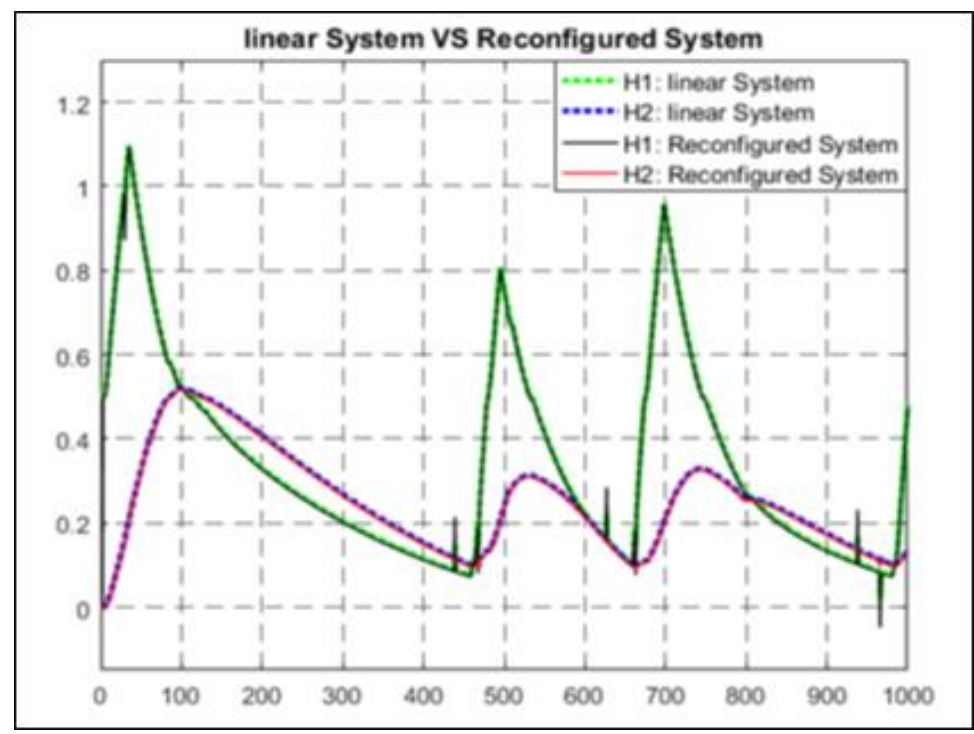

Figure 13. Comparison of the linear system with the reconfiguration system for a sensor fault 
Figure 14 is a Zoomed version of the igure 13; it shows that the effect of the faults on the system is compensated by the use of the adopted method.

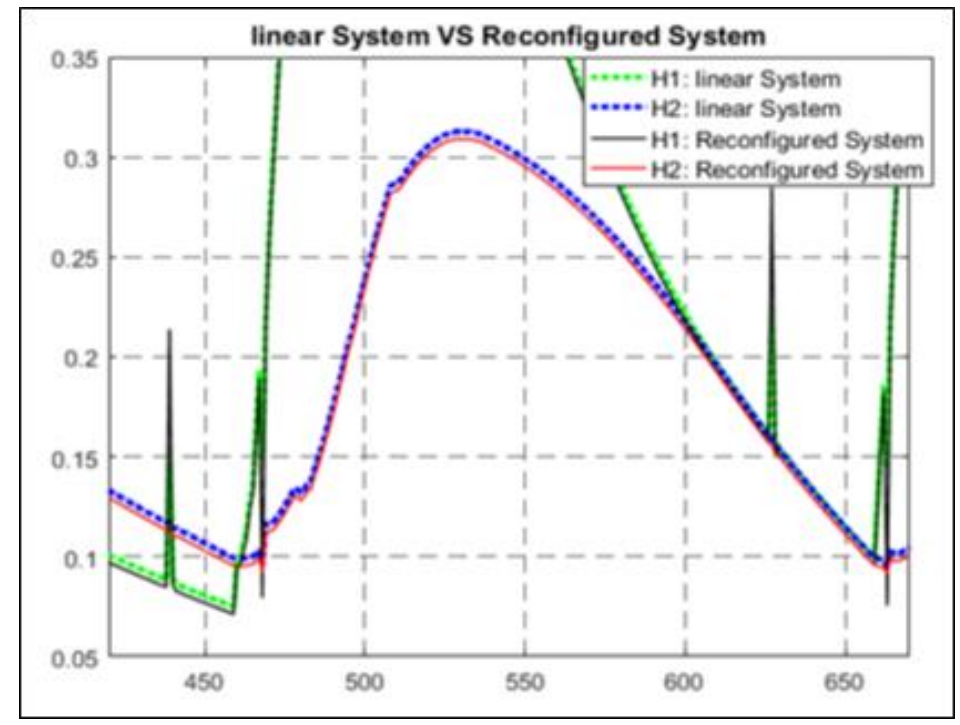

Figure 14. Comparison of the linear system with the reconfiguration system for a sensor fault (Zoom)

Figure 15 represents the error between the linear system and the reconfiguration system for a sensor fault; it shows the efficiency of the reconfiguration with Hammerstein method for the sensor fault system.

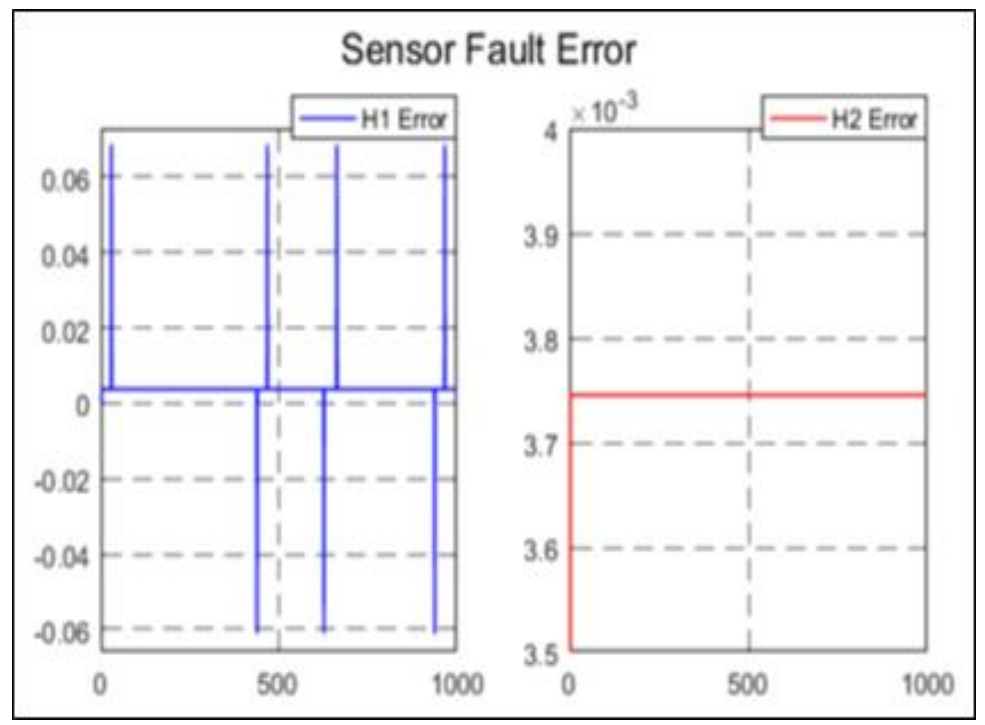

Figure 15. Error between the linear system and the reconfiguration system for a sensor fault

The computation of the error is realized for a time period between the linear system and the reconfiguration system for a sensor fault. It is interesting to note that the value of the error is around zero. From the previous figures, the Hammerstein method gives a very effective reconfiguration of the sensor faults.

Comparison between the reconfiguration methods for non linear hybrid systems and other methods: to prove the efficiency of the proposed reconfiguration method, an evaluation is given in this section. 
The result obtained using the Hammerstein method is compared to the methods developed in [16], [17] and summarized in the Table 2.

Table 2. Comparison of Methods

\begin{tabular}{lll}
\hline & Method Hammerstein & Method in [16], [17] \\
\hline Stability & $\begin{array}{l}\text { For each method, we try to compute the stability using from Lyapunov method } \\
\text { (Matlab LMI Control Toolbox). The methods guarantee the stabilily of the system. }\end{array}$ \\
$\begin{array}{ll}\text { Time for detected failure } \\
\begin{array}{l}\text { Feconfiguration } \\
\text { Re } \mathrm{T}=\text { few seconds) }\end{array}\end{array}$ & $\begin{array}{l}\text { Slow } \\
\text { the effect of the faults on the system is } \\
\text { compensated }\end{array}$ & $\begin{array}{l}\text { minimizes the propagation of failure } \\
\text { effects }\end{array}$ \\
\hline
\end{tabular}

The previous results show the effectiveness of the Hammerstein method for non linear hybrid systems. This approach ensuring a very important result to reconfiguration method (for the actuator faults and the sensor faults) and ensuring the stability of the systems.

\section{CONCLUSION}

During last years, there has been a lot researchs in the active fault tolerant control for non linear systems and linear Hybrid systems. [18], [19], [20], [21]. The contribution in this work is essentially to reformulate a new approach to improve the reconfiguration performance of the non linear hybrid system. This approch compensate the effect of faults and ensure the stability of the closed-loop system.

Therefore, this paper developed the linearization of a non linear Hybrid Systems using the Taylor method. Moreover, the active fault tolerant control based on the Hammerstein method has been proven to be efficient for the reconfiguration of the non linear Hybrid Systems in case of the intervention of the actuator fault and the sensor fault. The application of this method on the hydraulic system (two tanks with 4 modes) gives hopeful results. The future research will be focused on the reconfiguration method using the fault tolerant control for non linear hybrid systems based on neural network.

\section{ACKNOWLEDGEMENTS}

This work was not supported by any organization, there have been elaborated within the Laboratory of Research Analysis and Control of Systems (ACS) of ENIT, under the direction of Mme Moufida Ksouri.

\section{REFERENCES}

[1] Steinberg, M., "Historical overview of research in reconfigurable flight control.” Journal of Aerospace Engineerin., 219:263-275, 2005.

[2] Zhang, Y. et Jiang, J., "Bibliographical review on reconfigurable fault-tolerant control systems,” In Proc. of the 5th IFAC Symposium on Fault Detection, Supervision and safety for Technical Processes, June 9-11, Washington, D.C. USA. pages 265-276, 2003.

[3] C.Hanene , H.Yosra, K.Moufida, "Reconfiguration methods of PWA systems," 16th international conference on Sciences and Techniques of Automatic control \& computer engineering - STA'2015, Monastir, Tunisia, December 21-23, 2015.

[4] Ameur Sassi, Afef Abdelkrim, "New Stability Conditions for Nonlinear Systems Described by Multiple Model Approach," International Journal of Electrical and Computer Engineering (IJECE), Vol. 6, No. 1177 - 187, February 2016.

[5] H.Richter, W.P.M.H.Heemelsb, N.vande Wouwb, J. Lunzec, "Reconfigurable control of piecewise affine systems with actuator and sensor faults: Stability and tracking,” Automatica , 47,678-691, 2011.

[6] E. D. Sontag, “On the input-to-state stability property,” European J.Control, vol.1, pp.24-36, 1995.

[7] Thomas Nägele,Andrea Mair,Xiaoliang Sun. "Solving the Differential Biochemical Jacobian from Metabolomics Covariance Data,” PLoS ONE 9(4): e92299. Doi:10.1371/journal.pone.0092299, 2014.

[8] Paul Kirk1,2, Delphine M Y Rolando2, Adam L MacLean and Michael P H Stumpf , "Conditional random matrix ensembles and the stability of dynamical systems," Publishing Ltd and Deutsche Physikalische Gesellschaft, Published 13 August 2015.

[9] M. Duran Toksari, Yasemin Bilim, "Interactive Fuzzy Goal Programming Based on Jacobian Matrix to Solve Decentralized Bi-level Multi-objective Fractional Programming Problems,” Volume 17, pp 499-508, December 2015.

[10] Coralie Germain, Sébastien Briot1, Stéphane, Caro Natural, "Frequency Computation of Parallel Robots," J. Comput. Nonlinear Dynam 10(2), 021004, (11 pages) Paper No: CND-13-1194; doi: 10.1115/1.4028573, Mar 01, 2015.

[11] Xuan Zhao1, Jue Yang, Wenming Zhang1, Jun Zeng2, "Feedback Linearization Control for Path Tracking of Articulated Dump Truck," Telecommunication Computing Electronics and Control (TELKOMNIKA), Vol. 13, No. 3, 922 - 929, September 2015.

A Fault Tolerant Control for Sensor and Actuator Failures of a Non Linear Hybrid... (Chalandi Hanene) 
[12] P Sri Lakshmi*, V Lokesh Raju, “ECG De-noising using Hybrid Linearization Method,” Telecommunication Computing Electronics and Control (TELKOMNIKA), Vol. 15, No. 3, 504 - 508, September 2015.

[13] H.Richter, W.P.M.H.Heemelsb, N.vande Wouwb, J. Lunzec, "Reconfigurable control of piecewise affine systems with actuator and sensor faults: Stability and tracking,” Automatica, 678-691, 2011.

[14] Haili Zhang*, Hongli Zhang (2013), "Identification of Hammerstein Model Based on Quantum Genetic Algorithm," Telecommunication Computing Electronics and Control (TELKOMNIKA), Vol. 11, No. 12, 7206 - 7212, December 2013.

[15] J.Ben Slimane Dhifallah, K.Laabidi, M.Ksouri, “Support Vector Machines for Failures Diagnosis,” international Review of Automatic Control (I.RE.A.CO), Vol. 2, N.5, September 2009.

[16] S. Chaib, D. Boutat, A. Benali, F. Kratz, "Failure detection and reconstruction in switched nonlinear systems" Elsevier. Nonlinear Analysis: Hybrid Systems, 225_238, 2009.

[17] Panagiotis D. Christofides and Nael H. El-Farra, "Control of Nonlinear and Hybrid Process Systems: Designs for Uncertainty, Constraints and Time Delays,” Monograph - June 15, 2005.

[18] Zhang Dong-wen, Wang Qiao-ling, "Fault-Tolerant Control for a Class of Nonlinear Systems Based on Adaptive Observer,” Telecommunication Computing Electronics and Control (TELKOMNIKA), Vol.14, No.2A, pp. 324 334, June 2016.

[19] He Jinbao*1, Yi Xinhua2, Luo Zaifei3, Li Guojun4, "Backstepping Decentralized Fault Tolerant Control for Reconfigurable Modular Robots,” Telecommunication Computing Electronics and Control (TELKOMNIKA), Vol. 11, No. 7pp. $3508 \sim$ 3516, July 2013.

[20] Didier theilliol, Cédric join, Youmin zhang, "Actuator fault tolerant control design based on a reconfigurable reference input,” Int. J. Appl. Math. Comput. Sci., Vol. 18, No. 4, 553-560, 2008.

[21] Jiangang Lu*, Jie You, Qinmin Yang, "Nonlinear Model Predictive Controller Design for Identified Nonlinear Parameter Varying Model,” Telecommunication Computing Electronics and Control (TELKOMNIKA), Vol.10, No.3, pp. 514 523, September 2012. 